Check for updates

Cite this: Phys. Chem. Chem. Phys., 2019, 21, 26466

Received 28th October 2019 Accepted 1st November 2019 DOI: $10.1039 / \mathrm{c} 9 \mathrm{cp} 05839 \mathrm{~g}$ rsc.li/pccp

\section{Gelation enabled charge separation following visible light excitation using self-assembled perylene bisimides $\dagger$}

\author{
Charlotte L. Smith, ${ }^{a}$ Laura L. E. Mears, $\ddagger^{a}$ Benjamin J. Greeves, ${ }^{a}$ Emily R. Draper, (D) ${ }^{b}$ \\ James Doutch, ${ }^{\mathrm{C}}$ Dave J. Adams (D) ${ }^{\mathrm{b}}$ and Alexander J. Cowan (D) ${ }^{a}$
}

\section{Introduction}

PBIs are interesting materials for use in a range of solar energy technologies, including in organic photovoltaics, as photocatalysts, and as photoelectrodes for water splitting, ${ }^{1-6}$ due to their visible light harvesting properties, excellent thermal and electrochemical stabilities and ability to act as both electron donors and acceptors. A particularly attractive feature of PBIs is that they readily aggregate into structures consisting of $\pi$-stacked molecules, which can in principle enable both exciton and long-range charge transport through the structure. ${ }^{7-10}$ Designing functionality onto the PBIs that directs self-assembly offers a route to control the nature of the aggregates formed. This is important as changes in aggregate type, size or in the interlayer distance between the PBI units of the aggregate can have a profound effect on the photophysics and charge transport properties of the materials. ${ }^{2,10-12}$

\footnotetext{
${ }^{a}$ Stephenson Institute for Renewable Energy and the Department of Chemistry, University of Liverpool, Liverpool, L69 7ZF, UK. E-mail: acowan@liverpool.ac.uk

${ }^{b}$ School of Chemistry, College of Science and Engineering, University of Glasgow, Glasgow, G128QQ,UK. E-mail: dave.adams@glasgow.ac.uk

${ }^{c}$ STFC ISIS Neutron and Muon Source, Science and Technology Facilities Council, Rutherford Appleton Laboratory, Harwell Oxford, Didcot, OX11 OQX, UK

$\dagger$ Electronic supplementary information (ESI) available. See DOI: 10.1039/ c9cp05839g

\# Present address: Institute of Applied Physics, Vienna University of Technology, Vienna, 1040, Austria.
}

Recently a number of researchers, including some of us, ${ }^{1,13-15}$ have studied the photophysical properties and structures of PBIs functionalised at the imide sites with amino acids, Fig. 1a. A wide range of many different amino acid functionalised PBIs have now been synthesised, ${ }^{16}$ and it has been shown that these materials are also able to act as low molecular weight gelators (LMWGs). At $\mathrm{pH}$ values above those of the apparent $\mathrm{p} K_{\mathrm{a}}$ (or the lowest apparent $\mathrm{p} K_{\mathrm{a}}$ in cases where there are multiple values) of the pendant amino acid functionality, supramolecular structures form and are stable in solution even at very low $\left(<10^{-5} \mathrm{M}\right)$ concentrations. ${ }^{15}$ The self-assembled structures are typically long worm-like micelles, which consist of bundles of PBI fibres that persist on the micron length scale. ${ }^{16}$ At $\mathrm{pH}$ values below the apparent $\mathrm{p} K_{\mathrm{a}}$, hydrogel networks form which contain high concentrations of water within the films containing the self-assembled fibres. ${ }^{17}$ Early studies on dried solutions of the amino acid modified PBIs at high $\mathrm{pH}$, and on dried hydrogels (xerogels) demonstrated that the supramolecular structures formed gave rise to photoconductive materials. ${ }^{1,13}$ UV illumination leads to the formation of a radical anion $\left(\mathrm{PBI}^{\bullet-}\right)$ state that can then migrate through the supramolecular structure. ${ }^{1}$ Although these were highly promising results, particularly as the system was insensitive to $\mathrm{O}_{2}$, it was notable that the materials only became photoconductive under UV illumination, potentially limiting application.,16

In a previous study on alanine-functionalised PBI (PBI-A) using TA spectroscopy and DFT calculations, we demonstrated that the minimal activity under visible light was due to the lack 
(a)
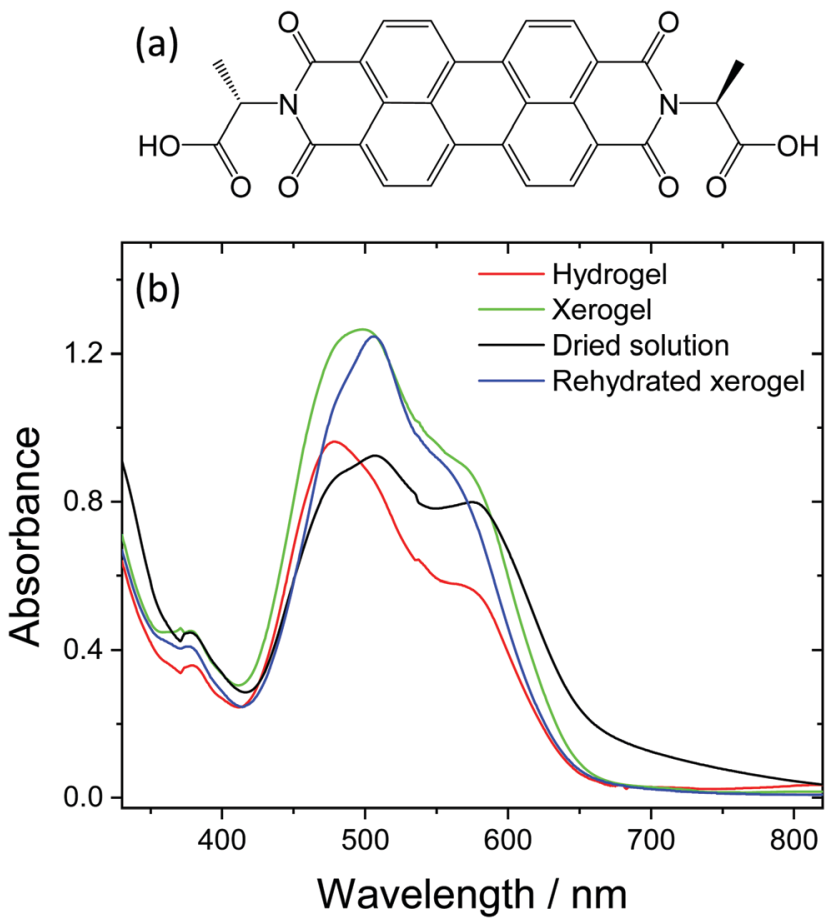

Fig. 1 Structure of PBI-A (a) and UV/Vis spectra of the films used within the study (dried solution, hydrogel, xerogel and rehydrated xerogel) (b). The hydrogel and rehydrated xerogel samples are immersed in $\mathrm{pH} 4$ aqueous solution to maintain hydration levels. All samples are prepared on conductive glass (FTO).

of charge separation (to form the $\mathrm{PBI}^{{ }^{-}}$state) in the low dielectric environment of the dried PBI-A film. ${ }^{15}$ Experiments and calculations demonstrated that the energy required to generate a charge separated state was greater than that available under visible illumination, and could only be achieved using higher energy UV photons. In contrast, visible light excitation led to charge separation and $\mathrm{PBI}^{\bullet^{-}}$formation when the PBI-A supramolecular structure was dissolved in water at high $\mathrm{pH}$ (9). The change in behaviour was assigned to a combination of the high dielectric constant of the aqueous solution $(\varepsilon \sim 80)$, which screens the free charge of the excess electron and hole and reduces the ion pair energy, and a calculated solvent induced change in the site of hole localisation. For PBI-A structures in aqueous solution, experimental electrochemical evidence and DFT calculations suggested that in the charge separated state the electron localised on the PBI core and the hole was localised on the alanine group. ${ }^{15}$ With dried films of PBI-A prepared at high pH (likely to exist as PBI- $\mathrm{A}^{2-}$ ), the site of electron accumulation was the same but it was predicted that the hole would partially localise on both the alanine (50\%) and the PBI core (50\%), placing it in closer proximity to the electron. Solvent controlled light-induced charge separation has been reported elsewhere with PBI based materials ${ }^{18,19}$ and it represents a potentially valuable way to achieve improved device activity. However, for use in many applications a solution-based absorber is undesirable. The generation of PBI-A hydrogels offers a route to forming self-supporting materials that contain the desired self-assembled structures whilst in a highly solvated environment, which may be beneficial for enabling formation of long-lived charge separated states, the formation of which is critical for solar energy conversion devices.

Hydrogels are also beginning to be explored for use as photocatalysts and photoelectrodes in water. ${ }^{20-23}$ Perylene monoimide gels have been studied as photocatalysts for hydrogen production in the presence of a sacrificial electron donor by the group of Stupp. ${ }^{20,21}$ Some of us have also reported the use of L-phenylalanine functionalised PBI (PBI-F) as a photocatalyst and in $\mathrm{pH}$ dependent studies shown that gelation at $\mathrm{pH} \sim 5$ correlates to a sudden increase in photocatalytic activity. ${ }^{22} \mathrm{~A}$ PBI-based organic photoelectrode for water oxidation has also been reported $^{24}$ and the current densities are amongst the highest achieved with an organic material. ${ }^{25,26}$ In that study, an aqueous solution of $N, N^{\prime}$-bis(phosphonomethyl)-3,4,9,10-perylenediimide (PMPDI) was spin-coated onto fluorine doped tin oxide (FTO) glass. Although the nature of the structures formed within the electrode were not described in detail, it is likely that by briefly dipping the films of dried PMPDI solution in acid, that either an outer gel layer was formed or that the entire structure was gelled. It is apparent that the development of highly active PBI hydrogel photocatalysts and photoelectrodes is feasible. These recent studies on light-driven water splitting further reinforce the need to develop an understanding of the factors controlling light driven charge separation within PBI hydrogel supramolecular structures. Here, we report a TA spectroscopic study of PBI-A dried solutions, hydrogels, air-dried hydrogels (xerogels) and rehydrated xerogels to explore the role of water content on charge separation yields and lifetime within the PBI supramolecular structures. Significantly, we demonstrate that self-supporting hydrogels and rehydrated xerogels are able to undergo visible light induced charge separation to form long-lived $\mathrm{PBI}^{\bullet}{ }^{-}$species, likely with the alanine acting as an electron donor, in a manner similar to the PBI-A structures in aqueous solution. The change in activity for the hydrated materials is proposed to be due to a decrease in the energy of the ion-pair state. The results for the hydrogel materials presented here have interesting wider implications for the design of organic photocatalysts and photoelectrodes. In contrast to inorganic materials, where effective screening of the mutual Coulomb attraction occurs enabling facile generation of free electron-hole pairs at room temperature, inefficiency of charge separation is commonly cited to be a limiting factor with many organic solar to fuels materials.

\section{Results}

Dried solution, hydrogel, xerogel and rehydrated xerogel PBI-A samples were prepared from a $7.5 \mathrm{mg} \mathrm{mL} \mathrm{mL}^{-1}$ PBI-A solution on FTO glass. Hydrogel and xerogel samples were prepared using a $\mathrm{HCl}$ vapour $\mathrm{pH}$ trigger and xerogels are dried in air for at least 17 hours. Rehydrated xerogels were soaked in $\mathrm{pH} 4$ solution for a minimum of 1 hour. Thermogravimetric measurements (Fig. S1, ESI $\dagger$ ) and room temperature mass loss experiments provide estimations of water contents for the different samples. As would be anticipated, the highest water content by weight is 
recorded for the hydrogel (hydrogel $~ 96 \%$, xerogel 5\%, rehydrated xerogel $\sim 72 \%$, dried solution $5 \%$ ).

We initially studied the UV/Vis spectra of the prepared PBI-A structures, Fig. 1. UV/Vis spectroscopy probes the electronic structure of the PBI-A structures and is sensitive to changes in the molecular level environment as the water content of the PBI-A sample is changed. In the visible region, a broad electronic transition $\left(S_{0}\right.$ to $\left.S_{1}\right)$ dominates, with a series of vibronic modes $\left(0-0^{\prime}, 0-1^{\prime}\right.$ and $\left.0-2^{\prime}\right)$ apparent between 400 and $650 \mathrm{~nm}$. In all cases, the spectra of the PBI films are broadened when compared to in solution at low concentration (Fig. S2, ESI $\dagger$ ), indicating extensive $\pi$-stacking and increased aggregation. A recent combined DFT, NMR and UV/Vis study of PBI-A at a range of $\mathrm{pH}$ values demonstrated that aggregates form in both solution and upon gelation. ${ }^{27}$ Furthermore the thin films formed by drying the deprotonated PBI-A solutions (referred to here as dried solutions) were predicted to contain similar structures to the hydrogel films but the structures would be present at different densities. In line with this, we observe that the dried solution, hydrogel, xerogel and rehydrated xerogel spectra are broadly similar, with only slight shifts in the UV/Vis maxima and the relative intensities of the vibronic modes. We therefore conclude that aggregated structures which contain extensive $\pi$-stacked PBI-A units are present in all the samples, regardless of water content. However, the small changes in the UV/Vis spectra, see in Fig. 1b, are still potentially significant as the changes in ratio of vibronic modes may indicate a different degree of overlap between PBI-A units within the structure, which could lead to a change in the supramolecular structure.

To further investigate the possible differences between the supramolecular structures of the gel materials as the water content is changed, small angle neutron scattering (SANS) measurements were carried out, Fig. 2 and Fig. S4-S7 (ESI $\dagger$ ). SANS can be applied to both wet and dry samples, the only requirement is a contrast in neutron scattering length density ${ }^{28}$ between the structures and their environment. The technique has been used extensively to study the nature of the aggregated structures within PBI materials on the nanometre to microns scale, thus complementing the UV/Vis spectroscopic data which addressed the molecular level environment. ${ }^{16,29}$ The SANS data could be fitted equally well to either a flexible elliptical cylinder model or a flexible cylinder combined with a power law. In brief, both of the flexible cylinder models are parameterised by the cylinder radius, the flexible segment (Kuhn) length and the contour length. Focussing first on the use of the flexible elliptical cylinder model, (Fig. 2) consistent with our previous data for the gel state, ${ }^{1,16}$ the fit to the model implies that there is little change in radius or axis ratio across the samples, with the radius being $2.5 \pm 0.1,3.1 \pm 0.3$ and $3.0 \pm 0.03 \mathrm{~nm}$ respectively and the axis ratio being $2.7,2.7$ and 2.8 respectively. The lengths are all very long as expected from the microscopy (Fig. S3, ESI $\dagger$ ). For the fit to the data using the flexible cylinder combined with a power law (Fig. 2 and Fig. S4-S6, ESI $\dagger$ ), drying of the hydrogel to form the xerogel apparently changes the primary fibre dimensions with the radius of fibres decreasing from $5.9 \pm 0.2 \mathrm{~nm}$ to $3.1 \pm 0.3 \mathrm{~nm}$.
Rehydration of the xerogel also led to partial, but not complete, recovery of the fibre radius $(5.0 \pm 0.5 \mathrm{~nm})$. A detailed discussion regarding the two model fits is available in the ESI, $\dagger$ but irrespective of the model used, the radii in all cases are greater than the molecular size and so SANS can be used to show the presence of long, anisotropic structures persisting across the hydrogel, xerogel and re-hydrated xerogel states.

As our structural characterisation (SANS, UV/Vis) measurements confirm the persistent presence of self-assembled aggregate structures, regardless of the water content of the PBI film, we have used TA spectroscopy to study if the changing water content of the sample changes the photophysical behaviour of the structures. The TA spectra recorded following $490 \mathrm{~nm}$ excitation of the PBI-A dried solution, hydrogel, xerogel and rehydrated xerogel samples are shown in Fig. 3 and 4. The TA spectra of the dried solution, Fig. 3(a) and 4(a), are markedly different from those of the hydrogel and the rehydrated xerogel samples, Fig. 4(b-d). The spectra recorded of the dried solution of PBI-A are very similar to those previously reported for a PBI-A dried solution prepared from a different concentration solution and are only briefly discussed..$^{15}$ Within 1 ps of excitation, broad positive absorptions centred at 740 and $840 \mathrm{~nm}$ are observed, which were previously assigned to the formation of a PBI excited state, Fig. $3(\mathrm{a}) \cdot{ }^{15}$ The new transient bands decay rapidly $\left(t_{50 \%}=3.5 \mathrm{ps}\right.$ at $740 \mathrm{~nm}$, Fig. S8, ESI $\left.\dagger\right)$ and by 3 ns only a weak TA signal is present with maxima at $746 \mathrm{~nm}(718(\mathrm{sh}))$ and ca. $860 \mathrm{~nm}$ (br.), Fig. 3a. Comparison of the TA data at $3 \mathrm{~ns}$ to the spectrum of electrochemically generated $\mathrm{PBI}^{{ }^{-}}{ }^{-} \mathrm{A}$ (Fig. 3a, $\lambda_{\max }=544,715$ and $805 \mathrm{~nm}$ ) and to the previously reported spectrum of photochemically generated $\mathrm{PBI}^{{ }^{-}}$-A following UV excitation of a dried solution (ca. 550, ca. 725 and $810 \mathrm{~nm})^{15}$ shows minimal agreement. The shoulder at $718 \mathrm{~nm}$ in the TA spectrum here may indicate the formation of a fraction of $\mathrm{PBI}^{{ }^{-}}$, however if present, the yield of $\mathrm{PBI}^{\bullet-}$ formed in the dried solution is low. In line with our past studies ${ }^{15}$ we find here the formation of a charge separated state is not the dominant process following visible light $(490 \mathrm{~nm})$ excitation of dried solutions of PBI-A.

Visible light excitation of the hydrogel gives rise to new positive bands within 1 ps at 543, 650, 722 and $814 \mathrm{~nm}$, Fig. 3b and $4 \mathrm{~b}$. The TA spectrum of the hydrogel recorded at $3 \mathrm{~ns}$ is similar to that observed at very early times ( $1 \mathrm{ps}$ shown) with strong bands remaining at 546, 730, $819 \mathrm{~nm}$ and a weak shoulder at $\sim 650 \mathrm{~nm}$, Fig. 3(b). A comparison of the spectrum of electrochemically generated reduced hydrogel $\left(\mathrm{PBI}^{{ }^{-}}{ }^{-} \mathrm{A}, \lambda_{\max }=544,731\right.$ and $820 \mathrm{~nm}$ ) and the TA spectrum recorded at 3 ns shows excellent agreement indicating that visible light can lead to the formation of a $\mathrm{PBI}^{\bullet-}$ state. Previously, it has been reported electron accumulation occurs on the perylene core with PBI-A materials and here we find the TA and spectroelectrochemical (SEC) spectra to be in good agreement with those studies, Fig. $3{ }^{15,16}$ At the latest time that could be studied with our apparatus $(3.2 \mathrm{~ns}),>10 \%$ of the signal assigned to the $\mathrm{PBI}^{\bullet-}$ state remains (Fig. S9, ESI $\dagger$ ), indicating that visible light excitation is able to lead to the formation of a long-lived reduced PBI within the hydrogel, a key step for solar energy conversion materials. 

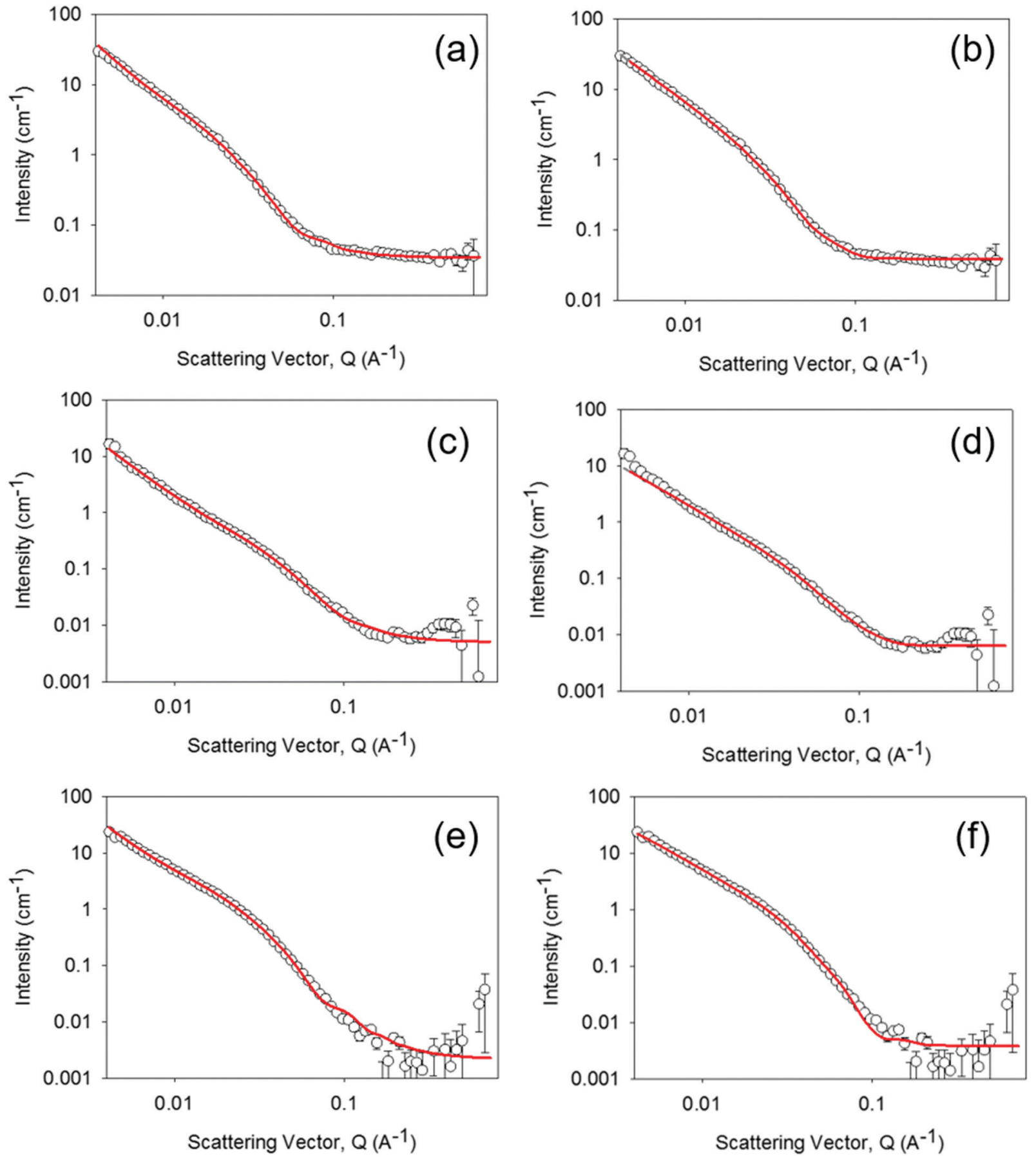

Fig. 2 SANS of $1 \mathrm{~mm}$ thick hydrogel (a and b), a xerogel prepared from a $1 \mathrm{~mm}$ thick hydrogel (c and d) and the rehydrated xerogel (e and f) following rehydration in $0.1 \mathrm{M} \mathrm{KCl}$ electrolyte, $\mathrm{pD} 4$, in $\mathrm{D}_{2} \mathrm{O}$ for 50 hours. Fit lines are shown in red and the data can be fitted to either a custom flexible cylinder combined with a power law model (a, $c$ and e) or a flexible elliptical cylinder model. All fitting data parameters and the relative contributions within the custom model are given in Tables S1, S2 and Fig. S4-S6 (ESI†). For the rehydrated xerogel sample of the remainder of the $1 \mathrm{~mm}$ demountable cell thickness was filled with $0.1 \mathrm{M} \mathrm{KCl}$ electrolyte in $\mathrm{D}_{2} \mathrm{O}$ pD 4 and the hydrogel was prepared in the same electrolyte.

The excellent agreement between the TA spectrum of the hydrogel at $3 \mathrm{~ns}$ and the SEC spectrum suggests that by $3 \mathrm{~ns}$ only the charge separated state is present, Fig. $3 \mathrm{~b}$. To further explore the formation of the charge separated state, a lifetime analysis has been carried out. In principle by approximating the kinetics of the transient absorption data to a small number of discreet exponential functions it is possible to carry out a global lifetime analysis that yields decay associated spectra and lifetimes can be carried out. However, the complex self-assembled structures studied here are shown below to give rise to a distribution of lifetimes making approximation to a small number of discreet exponential functions inappropriate. Instead, we have 


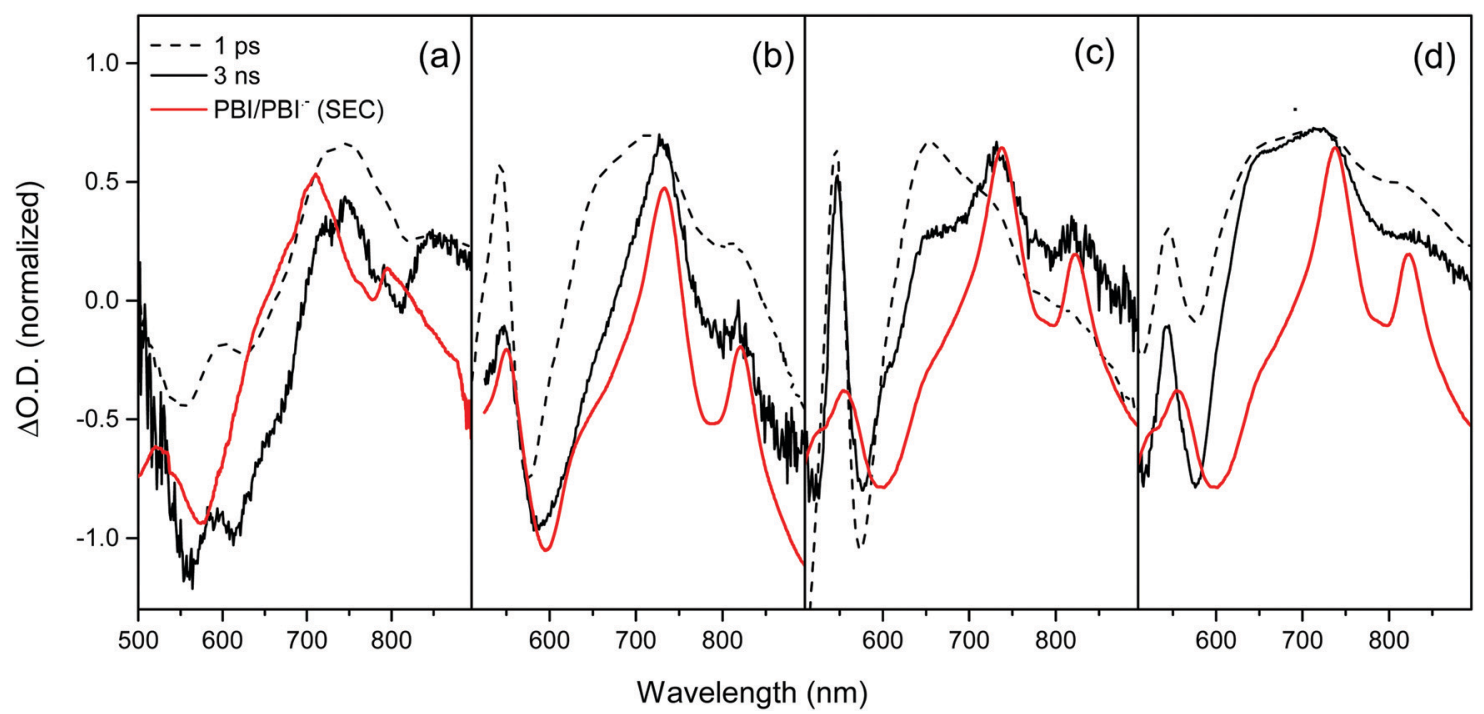

Fig. 3 Comparison between the TA spectra of PBI-A dried solution (a), hydrogel (b), rehydrated xerogel (c) and xerogel (d) recorded at 1 ps (dashed line) and 3 ns (solid line) following visible light excitation $(490 \mathrm{~nm})$ to the UV/Vis spectrum of electrochemically generated $\mathrm{PB} \mathbf{1}^{{ }^{-}-\mathrm{A}}\left(\mathrm{red}^{-}\right.$line).
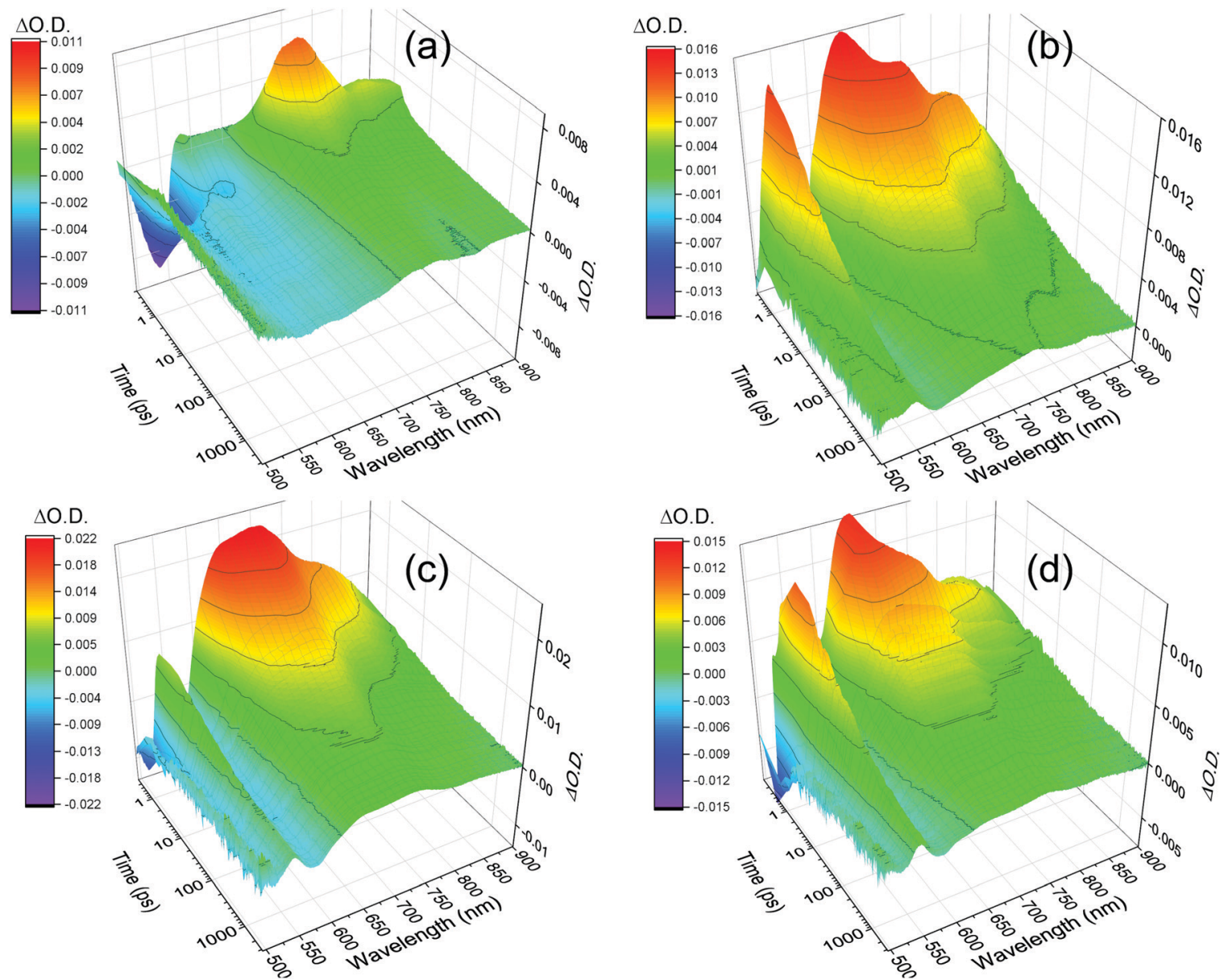

Fig. 4 TA spectra of PBI-A dried solution (a), hydrogel (b), xerogel (c) and rehydrated xerogel (d) recorded following visible light excitation (490 nm, $5 \mathrm{kHz}$ excitation repetition rate). All samples are on FTO glass. The hydrogel and rehydrated xerogel samples are immersed in argon purged pH 4 aqueous solution to maintain water content. All other samples are under an argon gas atmosphere. 


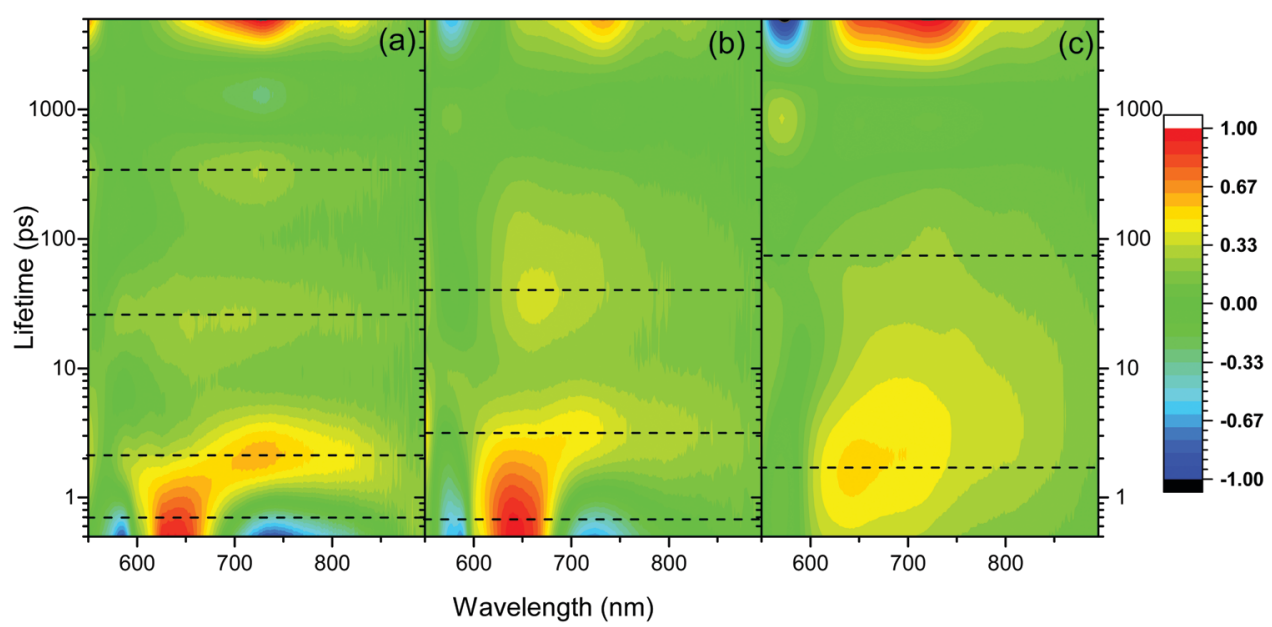

Fig. 5 Lifetime distribution maps (LDM) for PBI-A hydrogel (a), rehydrated xerogel (b) and xerogel (c) following visible light excitation (490 nm). The scale shows the relative pre-exponential amplitude of the decay function. Generated using a quasi-continuous distribution of 100 lifetimes over the timerange shown. The dotted lines indicate regions of interest discussed in the main text. 3D plots are shown in Fig. S10 (ESI†).

employed a lifetime density analysis (LDA) within the Opitmus ${ }^{30,31}$ data analysis suite. LDA uses a semi-continuous sum of exponents function to analyse transient kinetics without the need for an assumed model. ${ }^{31,32}$ The lifetime distribution maps (LDM) that are generated using 100 lifetimes between 0.3 and $5 \mathrm{~ns}$ are shown in Fig. 5. Full details of the fitting procedure, comparison of raw and fitted TA data from which the LDM (Fig. 5) is generated and the associated L-curves accompany Fig. S10-S14 (ESI $\dagger$ ). The LDM for the hydrogel shows that the initially generated feature at $650 \mathrm{~nm}$, likely due to formation of a PBI based singlet excited state, decays with lifetimes ranging from $\sim 0.4$ to 2 ps, Fig. $5 \mathrm{a}$. On these timescales we also observe decay lifetimes with a negative amplitude (indicative of an increase in optical density) at wavelengths associated with the $\mathrm{PBI}^{{ }^{-}}$state $($ca. 730 and $820 \mathrm{~nm}$ ) and the ground state $(580 \mathrm{~nm})$, Fig. 5(a). This suggests ultrafast charge separation and rapid formation of $\mathrm{PBI}^{{ }^{-}}$as the $650 \mathrm{~nm}$ excited state decays in the water rich hydrogel. The $\mathrm{PBI}^{\bullet-}$ state $(730,820 \mathrm{~nm})$ then decays over three main timescales. Initial rapid loss mechanisms have lifetimes distributed between 1.5 and 5 ps, with subsequent processes between 25 and 300 ps and at $>3 \mathrm{~ns}$. Interestingly, the LDM shows that a small population of the $650 \mathrm{~nm}$ band survives the initial fast decay processes and it then undergoes decay by mechanisms (likely through relaxation to the ground state based on the partial recovery at $580 \mathrm{~nm}$ ) with a distribution of lifetimes centred around 23 ps, Fig. 5 a.

The TA spectra of the rehydrated xerogel following $490 \mathrm{~nm}$ excitation are very similar to the hydrogel and at 1 ps new bands are present at 543, 650, 736 and $831 \mathrm{~nm}$, Fig. $3 \mathrm{c}$ and $4 \mathrm{~d}$. The TA spectrum at $3 \mathrm{~ns}$ shows maxima at 552,736 and $830 \mathrm{~nm}$ and it is in good agreement with the spectrum of electrochemically reduced PBI-A, Fig. 3c. A shoulder at $650 \mathrm{~nm}$ is still present in the TA spectrum at $3 \mathrm{~ns}$ and is more pronounced than in the electrochemically reduced sample. However, overall it is apparent that rapid formation of a $\mathrm{PBI}^{{ }^{-}}$based state can occur following visible light excitation of the rehydrated xerogel and that a significant population of this state remains at $>3 \mathrm{~ns}$.
The LDM of the rehydrated xerogel also shows behaviour similar to the hydrogel, Fig. 5b. The initially formed band at $650 \mathrm{~nm}$ undergoes a fast decay process with lifetimes between $\sim 0.4$ to $3 \mathrm{ps}$ as the bands assigned $\mathrm{PBI}^{\bullet-}$ increase, also indicating that ultrafast charge separation can occur in the rehydrated xerogel. The $650 \mathrm{~nm}$ band of $\mathrm{PBI}^{{ }^{-}}$decays with lifetimes over the 2 to 5 and 10 to 100 ps range. In line with the observation that a very small quantity of the initially formed excited state persists to the longest time-scales studied (3.2 ns), we also find that at $650 \mathrm{~nm}$ the LDA analysis generates lifetime components of around $4 \mathrm{~ns}$, Fig. $5 \mathrm{~b}$.

Analysis of the TA spectra recorded following visible light excitation of the xerogel is more complicated, Fig. $3 \mathrm{~d}$ and $4 \mathrm{c}$. It is not possible to carry out spectroelectrochemical studies of (dry) xerogels; as soon as they are placed in electrolyte they begin to rehydrate leading to structural changes. Therefore, we compare the TA data recorded to the spectroelectrochemical response of a rehydrated PBI-A xerogel as a best estimate $\left(\mathrm{PBI}^{\circ}{ }^{-}-\mathrm{A}, \lambda_{\max }=552,738,824 \mathrm{~nm}\right)$. The TA spectra of the xerogel shows bands at 542, 643, 721 and 833 (br.) $\mathrm{nm}$ in both the spectra recorded at 1 ps and $3 \mathrm{~ns}$, Fig. $3 \mathrm{~d}$. The TA bands observed at 542, 721 and 833 (br.) $\mathrm{nm}$ are assigned to the formation of a quantity of the $\mathrm{PBI}^{\circ}{ }^{-}$species, with the shift in maxima being due to solvation effects from the absence/low levels of water in the xerogel. However, it is unlikely that the strong band in the TA spectra at $643 \mathrm{~nm}$ at both $1 \mathrm{ps}$ and $3 \mathrm{~ns}$ is due to a $\mathrm{PBI}^{\bullet-}$ state on the basis of the spectroelectrochemical study. Therefore, the TA data demonstrates that at $3 \mathrm{~ns}$ the charge separated state is not the sole species present, and indicates that it is not the dominant, species. Compared to the rehydrated xerogel the efficiency of formation of a long-lived population of $\mathrm{PBI}^{{ }^{-}}$is significantly decreased in the dried xerogel.

The xerogel LDM is also markedly different to the hydrogel and rehydrated xerogel LDMs and offers insight into the lower yield of the $\mathrm{PBI}^{\bullet^{-}}$species at $3 \mathrm{~ns}$, Fig. 5(c). The initially formed excited state with a band at $\sim 643 \mathrm{~nm}$ is found to be stabilized 
in the xerogel and the distribution of initial decay lifetimes is $\sim 0.7$ to $7 \mathrm{ps}$, peaking at $2 \mathrm{ps}$, compared to $\sim 0.4$ to $2 \mathrm{ps}$ for the hydrogel. As the $643 \mathrm{~nm}$ state decays, the LDM does not show a clear negative amplitude for the decay components at longer wavelengths where $\mathrm{PBI}^{\bullet-}$ species would be expected to absorb (>700 nm). Instead, we only see evidence for the ground state recovery as indicated by the behaviour at $570 \mathrm{~nm}$, Fig. 5c. However, from the LDM we can identify distributions of decay lifetimes (20 to $200 \mathrm{ps}$ ) that are common at both $\sim 720$ and $815 \mathrm{~nm}$. This indicates that a small population of a $\mathrm{PBI}^{\bullet-}$ species is formed, likely with it being a minor pathway. This process occurs as the initial $643 \mathrm{~nm}$ band decays which would prevent the relatively small increase in optical density around 720 and $815 \mathrm{~nm}$ from being clearly observed. We therefore conclude that the lower yield of the charge separated state in the TA spectrum of the xerogel at $3 \mathrm{~ns}$ is due to a combination of both inefficient initial charge separation and significant levels of sub-ns recombination of those charges that do separate. Overall the TA spectroscopy shows the highest level of $\mathrm{PBI}^{\bullet-}$ formation following visible light excitation for the samples that contain the highest water content (hydrogel, rehydrated xerogel).

\section{Discussion}

UV/Vis spectroscopy confirms the persistent presence of aggregated PBI-A in all samples, regardless of the water content. However it is notable that the drying induced changes upon going from a hydrogel to a xerogel are not fully reversible, Fig. 1b. The UV/Vis spectrum of the rehydrated xerogel does not match that of the pristine hydrogel. The measurements of water content show that the rehydrated xerogel ( $72 \%$ water by weight) still contains less water than the pristine hydrogel (96\%). Therefore it is not surprising that UV/Vis spectroscopy, a probe of the electronic structure of the chromophore, which is sensitive to the local solvent-chromophore interactions, reports a difference between the spectra of the rehydrated xerogel and hydrogel. Significantly, SANS experiments demonstrate that the wider structural motifs are retained in all our PBI-A samples.

Therefore, we conclude that the differences in the TA spectra of the PBI-A samples upon drying are primarily due to solvation effects as the water content of the samples is changed. It is striking that the highest yields of long-lived $\mathrm{PBI}^{\bullet-}$ species and the fastest rates of charge separation following visible light excitation were measured with the water rich hydrogel sample. Furthermore, the lowest levels of long-lived charge separated states were measured in the samples with the lowest water content, the dried solution ( $5 \%$ water) and xerogel ( $5 \%$ water). Rehydration of the xerogel led to a recovery in the yield of charge separation, and in a manner similar to the hydrogel, $\mathrm{PBI}^{\bullet-}$ appears to dominate the TA spectrum at $3 \mathrm{~ns}$.

Experimentally, the change in Gibbs energy for photoinduced electron transfer $\left(\Delta G_{\mathrm{ET}}\right)$ in a donor-acceptor system can be estimated using eqn (1), providing a route to rationalising the observed TA trends. In eqn (1), $E_{\mathrm{OX}}$ and $E_{\mathrm{RED}}$ are the donor and acceptor oxidation and reduction potentials, $E^{*}$ the excited state energy, $C$ the coulombic interactions between the two ions and $S$ the difference in solvation energies between the sample under study and the solvent in which the oxidation and reduction potentials were measured.

$$
\Delta G_{\mathrm{ET}}=E_{\mathrm{OX}}-E_{\mathrm{RED}}-E^{*}-C+S
$$

Full details of the use of eqn (1) are given in the ESI $\dagger$ alongside Table S3, but its application requires a knowledge of the location of the donor and acceptor sites. The TA data provides evidence that visible light excitation of the hydrogel and rehydrated xerogel leads to the generation of high-yields of long-lived populations of $\mathrm{PBI}^{\bullet}$ - species (i.e., the electron acceptor site is a PBI core), but from these measurements alone we are unable to identify the spectral fingerprint of the electron donor. To explore the possible sites of electron donation, we have carried out a series of electrochemical measurements on gel electrodes in pH $40.1 \mathrm{M} \mathrm{KCl}$ electrolyte. Square wave voltammograms of the hydrogel and xerogel show the presence of an oxidation at $1.25 \mathrm{~V}$ and $1.15 \mathrm{~V}_{\mathrm{Ag} / \mathrm{AgCl}}$ respectively, in addition to features assignable to the reduction of PBI-A and $\mathrm{PBI}^{\bullet-}$-A, Fig. S15 and S16 (ESI $\dagger$ ). The energy of the transition between the lowest vibrational levels of the electronic ground and first excited states has been shown to be a suitable proxy for the electrochemical band gap of PBIs elsewhere. ${ }^{24}$ Based on these values $(2.17 \mathrm{eV}$ (hydrogel) and $2.13 \mathrm{eV}$ (rehydrated xerogel)) and the measured reduction potential (PBI-A/PBI ${ }^{\bullet-}$-A, $-0.46 \mathrm{~V}_{\mathrm{Ag} / \mathrm{AgCl}}$ (rehydrated xerogel) and -0.40 $\mathrm{V}_{\mathrm{Ag} / \mathrm{Agcl}}$ (hydrogel)), Fig. S15-S18, ESI $\dagger$ ), the oxidations at $1.25 \mathrm{~V}$ and $1.15 \mathrm{~V}_{\mathrm{Ag} / \mathrm{AgCl}}$ appear unlikely to be assignable to a PBI (core) based process. To further investigate the nature of these oxidations, we have carried out spectroelectrochemical measurements of the PBI-A hydrogel sample prepared on ITO coated $\mathrm{SrF}_{2}$, to provide an increased spectral window at shorter wavelengths, Fig. 6 (rehydrated xerogel, Fig. S19, ESI $\dagger$ ). Due to the cell design, it was necessary to utilise a silver pseudo reference electrode in these studies. Between 0.5 to $0.7 \mathrm{~V}_{\mathrm{Ag} / \mathrm{Ag}^{+}}$, the potential of the first oxidation that is reached, a decrease in optical density at $260 \mathrm{~nm}$ occurs and a new UV/Vis band forms at $295 \mathrm{~nm}$ (Fig. 6, inset). A slight decrease in the $S_{0}$ to $S_{1}$ bands at wavelengths between 400 and $650 \mathrm{~nm}$ also occurs at these potentials but no new positive bands are observed in this spectral region. As the potential of the hydrogel film is made more positive $(0.8 \mathrm{~V}$ to $1.1 \mathrm{~V}_{\mathrm{Ag} / \mathrm{Ag}^{+}}$), a new band is seen to grow in at $\sim 405 \mathrm{~nm}$ and a more pronounced bleach occurs at longer wavelengths, Fig. 6. Square-wave voltammetry also shows the presence of a 2nd oxidation at $\sim 1.4 \mathrm{~V}_{\mathrm{Ag} / \mathrm{AgCl}}, \sim 0.3 \mathrm{~V}$ positive of the alanine based oxidation. However, the proximity of the solvent window edge prevents accurate determination of the oxidation potential, Fig. S15 and S16 (ESI $\dagger$ ). The UV/Vis spectra of oxidised PBIs have been reported to have maxima across the visible region, $, 33,34$ including examples ${ }^{34}$ with bands between $400-450 \mathrm{~nm}$. Therefore the 2nd oxidation observed in the spectroelectrochemistry experiments at $405 \mathrm{~nm}$ is tentatively assigned to the oxidised species, $\mathrm{PBI}^{\bullet+}$-A. The UV/Vis feature at $260 \mathrm{~nm}$, present during the first oxidation, is in line with the spectrum of alanine which has been reported to have $\lambda_{\max }$ between 250 and $300 \mathrm{~nm} .{ }^{35}$ We therefore assign the first oxidation of the PBI-A hydrogel to an alanine based 


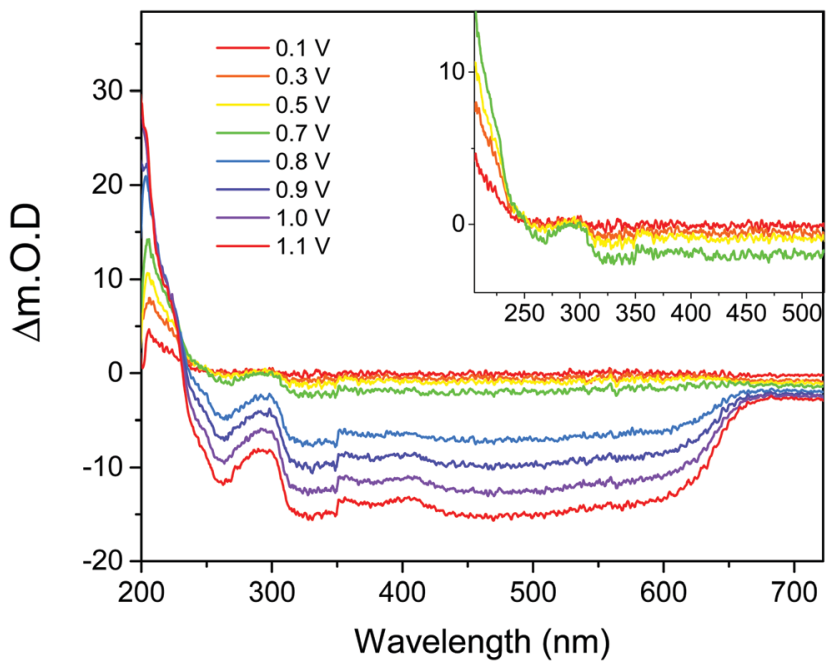

Fig. 6 Spectroelectrochemistry of the PBI-A hydrogel oxidations. Experiments are carried out at $\mathrm{pH} 4,0.1 \mathrm{M} \mathrm{KCl}$. The step at $350 \mathrm{~nm}$ is due to the change in light source and is an instrument artefact. Potential differences are reported versus a Ag wire pseudo reference electrode. The inset shows an expansion of the spectral changes occurring at the first oxidation.

process, Fig. 6. A similar response is seen for the rehydrated xerogel electrode, Fig. S18 (ESI $\dagger$ ). Therefore, on the balance of probability, we assign the electron donor site to be the alanine group, and the charge separated state in the TA experiments with hydrogel and rehydrated xerogel samples to be $\mathrm{PBI}^{\bullet-}-\mathrm{A}^{+}$. Using these electrochemically measured donor and acceptor potentials we estimate an ion-pair energy of $c a .1 .7 \mathrm{eV}$ for the hydrogel and ca. $1.6 \mathrm{eV}$ for the rehydrated xerogel. Therefore visible light excitation $(2.5 \mathrm{eV}, 490 \mathrm{~nm})$ is predicted sufficient to generate the charge separated state. Indeed we estimate a driving force $\left(\Delta G_{\mathrm{ET}}\right)$ of $c a$. $-0.55 \mathrm{eV}$ from the lowest energy singlet excited state with the water rich hydrogel and rehydrated xerogel samples, Table S3 (ESI $\dagger$ ).

As described above spectroelectrochemical studies on the dry xerogel are not possible; as soon as they are placed in electrolyte they begin to rehydrate. This has prevented us from experimentally determining the electron acceptor and donor sites. By assuming that the donor and acceptor sites remain the same as the hydrated samples we can estimate the effect of the change in dielectric environment on the Gibbs energy of the proposed photoinduced electron transfer, again using eqn (1). In this case we took the effective constant to be $\varepsilon \sim 3$, typical for a dried organic film. ${ }^{15}$ This gives rise to an estimated ion-pair energy of $c a .2 .2 \mathrm{eV}$, significantly greater than in the hydrated hydrogel and rehydrated xerogel samples, and no driving force $\left(\Delta G_{\mathrm{ET}} \sim 0.06 \mathrm{eV}\right)$ for charge separation following formation of the lowest energy singlet excited state. This result is readily understood as, in environments with a low dielectric permittivity, such as the dry xerogel, the excess free charge of the electron and hole are not effectively screened, increasing the ion pair energy. In contrast, in the water rich hydrogel (96\% water) and rehydrated xerogel (72\% water) PBI-A materials, the high dielectric permittivity of the environment enables screening of the excess free charge.
Our inability to carry out spectroelectrochemical measurements to assign the electron donor site in the dry xerogel means that the estimated Gibbs energy of photoinduced electron transfer needs to be treated with caution. Nonetheless the value of $c a .0 \mathrm{eV}$, arrived at assuming no change in the site of acceptor and donor sites upon film drying, is broadly in-line with our TA measurements where the level of $\mathrm{PBI}^{\bullet-}$ formed following $490 \mathrm{~nm}$ excitation is significantly less than measured with the hydrogel and rehydrated xerogel. Within eqn (1) the solvation term does not take into account specific solvent (water)-solute (PBI-A) interactions. Therefore, it is notable that eqn (1) successfully models the charge-separation results through considering just the change in the local dielectric constant upon hydration, without the need for specific PBI-A/water interactions. This result indicates that the effect of the water within the structure can be modelled as a bulk solvent that is charge screening and lowering the ion-pair energy. The formation of a small quantity of $\mathrm{PBI}^{\bullet-}$ within the xerogel, as measured by TA spectroscopy, is likely due to the small amount of residual water (5\%) within the PBI-A structure, which we did not account for within the calculation. The ion-pair energy for PBI-A dried solutions prepared at high pH (9) has been previously calculated to be very large, $c a .4 \mathrm{eV}$. Again this is in agreement with our TA results which shows that minimal charge separation occurs following visible light excitation. ${ }^{15}$ Therefore, the transient spectroscopic behaviour and the yield of visible light generated $\mathrm{PBI}^{\bullet-}$ can be largely rationalised by the presence or absence of water within the PBI-A structures.

\section{Conclusions}

Self-assembled PBI-A films can be prepared in a variety of structures and hydration levels, which makes them an ideal model material to examine the effect of hydration on charge separation yields and kinetics. Transient UV/Vis, electrochemical and spectroelectrochemical measurements show that PBI-A hydrogels can undergo visible light induced charge separation with the alanine acting as an electron donor and the PBI core as an electron acceptor. It is clear that in the water rich hydrogels high yields of long-lived charge separated states can be generated following visible light excitation. In contrast, dried films of solution and the dried hydrogel films (xerogels) of PBI-A show lower levels of charge separation under visible illumination. This result is rationalised by the high dielectric permittivity of the aqueous environment within the hydrated samples that facilitates both the formation of and stabilisation of the charge separated state. The observed facile charge separation in the hydrated samples is important, as a significant issue with many organic photocatalysts and photoelectrodes is that the low dielectric environment of the bulk polymer leads to generation of free charges only occurring at defect sites ${ }^{36}$ or at the polymer/ water (or electron donor) interface. ${ }^{37,38}$ Therefore, these results coupled to the emerging reports of high levels of photocatalytic activity with similar hydrogel materials ${ }^{20,22,24}$ indicates that the development of water rich-hydrogel materials is a promising 
approach to generating useful materials for the production of solar fuels.

\section{Experimental methods}

\section{Materials}

Milli-Q water (18.2 M $\Omega$ ) was used throughout (Millipore Corp). All chemicals were obtained from Sigma-Aldrich and used as received, except for PBI-A which was synthesised according to literature procedures. ${ }^{1}$ Argon and nitrogen were purchased from BOC at pureshield grade.

\section{Preparation of PBI-A gel electrodes}

FTO (TEC-15) substrates were cleaned by sonication for 20 minutes in three different solutions; $1 \%$ Helmanex solution, ethanol and water, and rinsed with water between each sonication. After drying of the FTO glass substrates with compressed air, they were heated in an oven to $400{ }^{\circ} \mathrm{C}\left(20^{\circ} \mathrm{C} \mathrm{min}^{-1}\right)$ and held for 20 minutes before cooling. Quick drying epoxy resin was used to mask out a surface area of $150 \mathrm{~mm}^{2}\left(10 \times 15 \mathrm{~mm}^{2}\right)$. Gelation was carried out in a Petri dish within a humidified glove bag in order to ensure PBI-A gel electrodes did not dry out. FTO glass substrates were placed in a Petri dish containing 8 layers of blue roll dampened with water, and $20 \mu \mathrm{L}$ of PBI-A $\left(7.5 \mathrm{mg} \mathrm{mL} \mathrm{mL}^{-1}\right.$ dissolved with 1 molar equivalence of $0.1 \mathrm{M} \mathrm{NaOH}$ with the remainder made up with Milli-Q water) was deposited onto each FTO conductive surface. 40 drops of concentrated $\mathrm{HCl}$ were evenly dropped around both the outer corners of the Petri dish and near to the PBI-A FTO electrode to lower the $\mathrm{pH}$. The lid was placed on the Petri dish and sealed with parafilm, allowing for an acidic atmosphere to build up within the Petri dish. Typically, a gel was formed after around $c a$. 20-40 minutes. Once gelled, the PBI-A electrode was transferred out of the Petri dish into a vial containing $0.1 \mathrm{M} \mathrm{KCl}$ electrolyte ( $\mathrm{pH} 4$ adjusted by $1 \mathrm{M} \mathrm{HCl}$ ) in the glove bag to prevent drying. In the case of PBI-A xerogel electrodes, the electrode was removed from the glove bag, and allowed to dry in air for at least 17 hours. By this time only minimal change in water content occurs, consistent with previous reports (albeit at a slightly lower concentration of PBI-A). ${ }^{1}$ Rehydrated xerogels are defined as xerogels which are left to rehydrate in $0.1 \mathrm{M} \mathrm{KCl} \mathrm{pH} 4$ electrolyte for between 1-4 hours. The exception to this is for SANS experiments where the timescale of the measurement is longer and, in this case, the samples were left to rehydrate for 50 hours prior to the start of the experiment. Water contents for the xerogel and dry solution were obtained by TGA (thermogravimetric analysis) (Fig. S1, ESI $\dagger$ ) with samples studied between 20 and $200{ }^{\circ} \mathrm{C}$ and held at $120{ }^{\circ} \mathrm{C}$ for 20 minutes. The hydrogel and rehydrated xerogel samples were found to dry immediately upon exposure to air and the transfer time from the FTO to the TGA apparatus prevented reliable water content data from being obtained in this manner. Instead water contents of hydrogel and rehydrated xerogel samples were measured by room temperature mass loss in air. Samples were placed directly onto a balance, surface water removed, and the mass monitored for 24 hours.

\section{Femtosecond transient absorption (TA) spectroscopy}

TA spectroscopy was carried out using a HELIOS spectrometer (Ultrafast Systems) PHAROS laser (Light Conversion Ltd) operating at $10 \mathrm{kHz}$ coupled to an ORPHEUS optical parametric amplifier (Light Conversion Ltd) in tandem with a LYRA harmonic generator (Light Conversion Ltd). The system has been described in detail elsewhere. ${ }^{15}$ The pump wavelength was $490 \mathrm{~nm}(275 \mu \mathrm{W})$ with samples excited at $5 \mathrm{kHz}$. Thin film PBI-A $(20 \mu \mathrm{L}$ PBI-A, $7.5 \mathrm{mg} \mathrm{mL}^{-1}$, gelled over $150 \mathrm{~mm}^{2}$ area on FTO) hydrogel and partially rehydrated xerogel samples were recorded in a $2 \times 1 \mathrm{~cm}^{2}$ quartz cuvette containing $\mathrm{KCl}$ electrolyte $(0.1 \mathrm{M} \mathrm{KCl}, \mathrm{pH} 4$ adjusted by $1 \mathrm{M} \mathrm{HCl}$ ). LDM analysis was carried out using the module within the OPITMUS software. ${ }^{31}$

\section{UV/Vis spectroscopy, electrochemistry, spectroelectrochemistry}

UV/Vis spectroscopy was carried out using a Shimadzu UV-2600 spectrophotometer. To study the reduced PBI-A samples a $2 \times 1 \mathrm{~cm}^{2}$ quartz cell was used containing a $5 \mu \mathrm{L}$ PBI-A FTO electrode, reference and counter electrodes in $\mathrm{KCl}$ electrolyte (0.1 M, pH 4 adjusted with $1 \mathrm{M} \mathrm{HCl}$ ), spectra were recorded at $0.1 \mathrm{~V}$ steps negative from open circuit potential, data shown in Fig. 4 is for the first reductive process observed. To study the PBI-A oxidations an increased spectral window was needed therefore experiments were carried out using ITO coated $\mathrm{SrF}_{2}$ windows coated with PBI-A. Samples were placed within a Harrick cell with $1 \mathrm{~mm}$ pathlength with $\mathrm{Ag}$ and Pt wires placed close to the PBI-A electrode as the pseudo reference and counter electrode respectively.

Electrochemical measurements were carried out in a custom-designed 3 electrode cell. The working electrode was the PBI-A sample on an FTO glass substrate connected via a crocodile clip and nickel wire. The reference electrode was $\mathrm{Ag} / \mathrm{AgCl}(3.5 \mathrm{M} \mathrm{KCl})$, a counter electrode of $\mathrm{Pt}$ wire, and $\mathrm{KCl}$ electrolyte $(0.1 \mathrm{M}, \mathrm{pH} 4$ adjusted by $\mathrm{HCl})$ were used in all experiments. Palmsens potentiostat was used to carry out electrochemical methods. Prior to all experiments the cell was degassed under a stream of argon. Square wave voltammetry was performed under a nitrogen atmosphere at a frequency of $25 \mathrm{~Hz}$ with an equilibration time of 90 or 120 seconds. During DCVA measurements the change in transmission of the PBI-A sample is measured against time with a Shimadzu UV-2600 spectrophotometer. Transmission versus time measurements were carried out at $487 \mathrm{~nm}, 608 \mathrm{~nm}, 729 \mathrm{~nm}$ and $820 \mathrm{~nm}$ whilst running a $\mathrm{CV}$ at $100 \mathrm{mV} \mathrm{s}^{-1}$ between $+1.4 \mathrm{~V}$ to $-0.8 \mathrm{~V}_{\mathrm{Ag} / \mathrm{AgCl}}$.

\section{SEM}

SEM was carried out using an Hitachi S-4800 FE-SEM. Silver conductive paste was used to adhere the FTO/PBI-A electrode to the surface of an SEM stub, and silver paste was coated around the top and outer edges of the electrode to create a connection to the SEM stub. Samples were measured in deceleration mode with an acceleration voltage of $1.5 \mathrm{kV}$, using an upper electron detector, and a working distance of $3 \mathrm{~mm}$. Due to a large build-up of charge the SEM had to be taken immediately after focusing. 


\section{Small angle neutron scattering (SANS)}

SANS measurements were carried out on the SANS2D instrument at the STFC ISIS Pulsed Neutron Source (Didcot, UK). PBI-A solutions were prepared at $7.5 \mathrm{mg} \mathrm{mL}^{-1}$ as above, except $\mathrm{D}_{2} \mathrm{O}$ and $\mathrm{NaOD}$ were used. Gels were prepared on quartz discs with a $1 \mathrm{~mm}$ PTFE spacer creating a well for the gel. Here, the gels were thicker compared to that used for previous experiments (specifically $240 \mu \mathrm{L}$ over $113 \mathrm{~mm}^{2}$ ), and were required to fill the cell and obtain a suitable signal from the scattering and were gelled as above using DCl vapour. Hydrated gels were covered with a second quartz disc before loading into aluminum cells, as were the xerogels once dried and the rehydrated xerogels were loaded into the same cells with the $1 \mathrm{~mm}$ space filled with $\mathrm{KCl}$ electrolyte $(0.1 \mathrm{M}, \mathrm{pD} 4)$ made in $\mathrm{D}_{2} \mathrm{O}$. All cells were housed in a temperature-controlled sample rack at $25{ }^{\circ} \mathrm{C}$ during the measurements. The datasets were background subtracted with the empty quartz cell and $\mathrm{D}_{2} \mathrm{O}$ as appropriate. Where necessary the amount of $\mathrm{D}_{2} \mathrm{O}$ background to be subtracted was scaled according to the transmission to account for small variations in cell spacer thickness.

All raw analytical is freely available from the University of Liverpool data catalogue and can be found at http://datacat. liverpool.ac.uk/.

\section{Conflicts of interest}

There are no conflicts to declare.

\section{Acknowledgements}

The UK Central Laser Facility (STFC) Laser Loan Pool is acknowledged for access to the TA spectrometer (EP/G03088X/1). AC, DA, BG acknowledge the Leverhulme Trust for funding (RPG-2018-013). Dr M. Forster and Dr K. Arnold are thanked for help with SEM measurements and Dr J. Lee for assistance with TA measurements. Prof. T. Veal is thanked for the ITO coated $\mathrm{SrF}_{2}$. DA thanks the EPSRC for a Fellowship (EP/L021978/1). ED thanks the Leverhulme Trust for funding (ECF-2017-223) and the University of Glasgow for an LKAS Leadership Fellowship. Experiments at the ISIS Pulsed Neutron and Muon Source were supported by a beamtime allocation (RB1720272) from the Science and Technology Facilities Council. This work benefitted from SasView software, originally developed by the DANSE project under NSF award DMR-0520547.

\section{References}

1 E. R. Draper, J. J. Walsh, T. O. McDonald, M. A. Zwijnenburg, P. J. Cameron, A. J. Cowan and D. J. Adams, J. Mater. Chem. C, 2014, 2, 5570-5575.

2 F. Würthner, Chem. Commun., 2004, 1564-1579.

3 D. Sun, D. Meng, Y. Cai, B. Fan, Y. Li, W. Jiang, L. Huo, Y. Sun and Z. Wang, J. Am. Chem. Soc., 2015, 137, 11156-11162.

4 R. Marty, R. Nigon, D. Leite and H. Frauenrath, J. Am. Chem. Soc., 2014, 136, 3919-3927.
5 S. Wolter, K. Magnus Westphal, M. Hempel, F. Würthner, O. Kühn and S. Lochbrunner, J. Chem. Phys., 2017, 50, 184005.

6 R. J. Lindquist, B. T. Phelan, A. Reynal, E. A. Margulies, L. E. Shoer, J. R. Durrant and M. R. Wasielewski, J. Mater. Chem. A, 2016, 4, 2880-2893.

7 P. E. Hartnett, E. A. Margulies, H. S. S. R. Matte, M. C. Hersam, T. J. Marks and M. R. Wasielewski, Chem. Mater., 2016, 28, 3928-3936.

8 S. Berardi, V. Cristino, M. Canton, R. Boaretto, R. Argazzi, E. Benazzi, L. Ganzer, R. Borrego Varillas, G. Cerullo, Z. Syrgiannis, F. Rigodanza, M. Prato, C. A. Bignozzi and S. Caramori, J. Phys. Chem. C, 2017, 121(33), 17737-17745.

9 H. Marciniak, X. Q. Li, F. Würthner and S. Lochbrunner, J. Phys. Chem. A, 2011, 115, 648-654.

10 F. Würthner, C. R. Saha-Möller, B. Fimmel, S. Ogi, P. Leowanawat and D. Schmidt, Chem. Rev., 2016, 116, 962-1052.

11 M. Más-Montoya and R. A. J. Janssen, Adv. Funct. Mater., 2017, 27, 1605779.

12 K. M. Lefler, D. T. Co and M. R. Wasielewski, J. Phys. Chem. Lett., 2012, 3, 3798-3805.

13 S. Roy, D. Kumar Maiti, S. Panigrahi, D. Basak and A. Banerjee, RSC Adv., 2012, 2, 11053.

14 E. R. Draper and D. J. Adams, Chem, 2017, 3, 390-410.

15 J. J. Walsh, J. R. Lee, E. R. Draper, S. M. King, F. Jackel, M. A. Zwijnenburg, D. J. Adams and A. J. Cowan, J. Phys. Chem. C, 2016, 120, 18479-18486.

16 E. R. Draper, L. J. Archibald, M. C. Nolan, R. Schweins, M. A. Zwijnenburg, S. Sproules and D. J. Adams, Chem. Eur. J., 2018, 24, 4006-4010.

17 J. Raeburn, A. Zamith Cardoso and D. J. Adams, Chem. Soc. Rev., 2013, 42, 5143.

18 A. Nowak-Król, B. Fimmel, M. Son, D. Kim and F. Würthner, Faraday Discuss., 2015, 185, 507-527.

19 Y. Wu, R. M. Young, M. Frasconi, S. T. Schneebeli, P. Spenst, D. M. Gardner, K. E. Brown, F. Würthner, J. F. Stoddart and M. R. Wasielewski, J. Am. Chem. Soc., 2015, 137, 13236-13239.

20 A. S. Weingarten, R. V. Kazantsev, L. C. Palmer, M. McClendon, A. R. Koltonow, A. P. S. Samuel, D. J. Kiebala, M. R. Wasielewski and S. I. Stupp, Nat. Chem., 2014, 6, 964-970.

21 A. S. Weingarten, R. V. Kazantsev, L. C. Palmer, D. J. Fairfield, A. R. Koltonow and S. I. Stupp, J. Am. Chem. Soc., 2015, 137, 15241-15246.

22 M. C. Nolan, J. J. Walsh, L. L. E. Mears, E. R. Draper, M. Wallace, M. Barrow, B. Dietrich, S. M. King, A. J. Cowan and D. J. Adams, J. Mater. Chem. A, 2017, 5, 7555-7563.

23 J. Yang, H. Miao, Y. Wei, W. Li and Y. Zhu, Appl. Catal., B, 2019, 240, 225-233.

24 J. T. Kirner, J. J. Stracke, B. A. Gregg and R. G. Finke, ACS Appl. Mater. Interfaces, 2014, 6, 13367-13377.

25 J. T. Kirner and R. G. Finke, J. Mater. Chem. A, 2017, 5, 19560-19592.

26 L. Yao, A. Rahmanudin, N. Guijarro and K. Sivula, Adv. Energy Mater., 2018, 8, 1802585.

27 E. R. Draper, L. Wilbraham, D. J. Adams, M. Wallace and M. Zwijnenburg, Nanoscale, 2019, 11, 15917-15928. 
28 L. L. E. Mears, E. R. Draper, A. M. Castilla, H. Su, B. Dietrich Zhuola, M. C. Nolan, G. N. Smith, J. Doutch, S. Rogers, R. Akhtar, H. Cui and D. J. Adams, Biomacromolecules, 2017, 18, 3531-3540.

29 E. R. Draper, B. J. Greeves, M. Barrow, R. Schweins, M. A. Zwijnenburg and D. J. Adams, Chem, 2017, 2, 716-731. 30 www.sasview.org.

31 C. Slavov, H. Hartmann and J. Wachtveitl, Anal. Chem., 2015, 87, 2328-2336.

32 G. F. Dorlhiac, C. Fare and J. J. van Thor, PLoS Comput. Biol., 2017, 13, e1005528.

33 F. Würthner, A. Sautter, D. Schmid and P. J. Weber, Chem. Eur. J., 2001, 7, 894-902.
34 F. Ronconi, Z. Syrgiannis, A. Bonasera, M. Prato, R. Argazzi, S. Caramori, V. Cristino and C. A. Bignozzi, J. Am. Chem. Soc., 2015, 137, 4630-4633.

35 X. Li, K. Xu, H. Li, S. Yao, Y. Li and B. Liang, RSC Adv., 2016, 6, 8395-8405.

36 V. S. Vyas, F. Haase, L. Stegbauer, G. Savasci, F. Podjaski, C. Ochsenfeld and B. V. Lotsch, Nat. Commun., 2015, 6, 8508.

37 M. Sachs, R. S. Sprick, D. Pearce, S. A. J. Hillman, A. Monti, A. A. Y. Guilbert, N. J. Brownbill, S. Dimitrov, X. Shi, F. Blanc, M. A. Zwijnenburg, J. Nelson, J. R. Durrant and A. I. Cooper, Nat. Commun., 2018, 9, 4968.

38 D. J. Woods, R. S. Sprick, C. L. Smith, A. J. Cowan and A. I. Cooper, Adv. Energy Mater., 2017, 7, 1700479. 